ARTICLE

DOI: $10.1038 / \mathrm{s} 41467-018-03041-1$

\title{
The role of confined collagen geometry in decreasing nucleation energy barriers to intrafibrillar mineralization
}

\author{
Doyoon Kim (1) ${ }^{1}$, Byeongdu Lee (10) ${ }^{2}$, Stavros Thomopoulos ${ }^{3} \&$ Young-Shin Jun (1) ${ }^{1}$
}

Mineralization of collagen is critical for the mechanical functions of bones and teeth. Calcium phosphate nucleation in collagenous structures follows distinctly different patterns in highly confined gap regions (nanoscale confinement) than in less confined extrafibrillar spaces (microscale confinement). Although the mechanism(s) driving these differences are still largely unknown, differences in the free energy for nucleation may explain these two mineralization behaviors. Here, we report on experimentally obtained nucleation energy barriers to intra- and extrafibrillar mineralization, using in situ X-ray scattering observations and classical nucleation theory. Polyaspartic acid, an extrafibrillar nucleation inhibitor, increases interfacial energies between nuclei and mineralization fluids. In contrast, the confined gap spaces inside collagen fibrils lower the energy barrier by reducing the reactive surface area of nuclei, decreasing the surface energy penalty. The confined gap geometry, therefore, guides the two-dimensional morphology and structure of bioapatite and changes the nucleation pathway by reducing the total energy barrier.

\footnotetext{
${ }^{1}$ Department of Energy, Environmental \& Chemical Engineering, Washington University in St. Louis, St. Louis, MO 63130, USA. ${ }^{2}$ X-ray Science Division, Argonne National Laboratory, Argonne, IL 60439, USA. ${ }^{3}$ Department of Orthopedic Surgery, Columbia University, New York, NY 10032, USA.

Correspondence and requests for materials should be addressed to Y.-S.J. (email: ysjun@seas.wustl.edu)
} 
T he nucleation and growth of mineral phases in porous media are critical for many biologic processes and engineering applications ${ }^{1-4}$. Recent studies have investigated nucleation in confined nanoscale pore spaces ${ }^{1,5,6}$, where the physicochemical properties, such as the melting point or crystal polymorphism ${ }^{6}$, of the minerals formed in the pores are clearly distinct from their bulk phase counterparts. These findings provide a better understanding of biominerals. For example, hydroxyapatite (HA) crystals nucleated in the $25-300 \mathrm{~nm}$ pores showed stronger orientation than in bulk solution ${ }^{7}$, as commonly observed in bioapatite (a biologically produced analog of HA) in bones and teeth ${ }^{8}$. However, we know little about how nanoscale confinement affects the nucleation of calcium phosphate minerals $(\mathrm{CaP})$ in more physiologically relevant systems.

Mineralization of the skeleton relies on the nucleation and growth of mineral crystals in both unconfined and confined spaces $^{7,9,10}$. CaP mineralization takes place in nanoscale porous structures created by a unique arrangement of collagen molecules $^{11-13}$. Narrow channel-like gap regions $(\sim 40 \mathrm{~nm}$ long and $\sim 20 \mathrm{~nm}$ high $)^{11,14-16}$ in type I collagen molecules are known to provide appropriate spaces for intrafibrillar mineralization (IM), forming oriented bioapatite ${ }^{11,17,18}$. Thus, in addition to noncollagenous proteins (NCPs) in the extracellular matrix ${ }^{15,17,19}$, the collagen fibrillar structure itself has been emphasized as another major factor in IM. Recently, Wang et al. reported bonelike bioapatite formation in collagen in vitro within a collagen structure during fibrillogenesis, even without $\mathrm{NCPs}^{10}$. Nudelman et al. showed that a specific band position in the gap region of collagen with a net positive charge can attract net negatively charged amorphous calcium phosphate (ACP) nuclei in the presence of polyaspartic acid (pAsp), which is a well-known substitute for NCPs in biomimetic experiments ${ }^{20}$.

Bioapatite crystals are also found in the unconfined extrafibrillar spaces of collagen (extrafibrillar mineralization, EM) as aggregate without a specific orientation ${ }^{21,22}$. Our previous work showed that the pathways and kinetics of bioapatite formation during IM and EM were distinct from each other ${ }^{23}$. The nucleation pathway for EM included aggregation and densification of prenucleation clusters to form spherical ACP as an intermediate product. A similar pathway was reported to occur in biomimetic environments, but without confinement ${ }^{24-26}$. On the other hand, such an intermediate stage did not appear in the IM pathway for in vitro collagen mineralization, suggesting a direct formation of plate-like particles, as reported in our previous study $^{23}$. Despite the absence of an intermediate step, the kinetics for IM were, however, slower than for EM, due to the nucleationinhibiting effect of $\mathrm{pAsp}^{23,25}$.

Despite experimental evidence of different nucleation patterns in IM and to EM, the mechanism(s) behind these processes remain unclear. The differences in nucleation pathways and kinetics imply that the nucleation energy barriers differ in EM and IM. For example, the formation of prenucleation clusters,

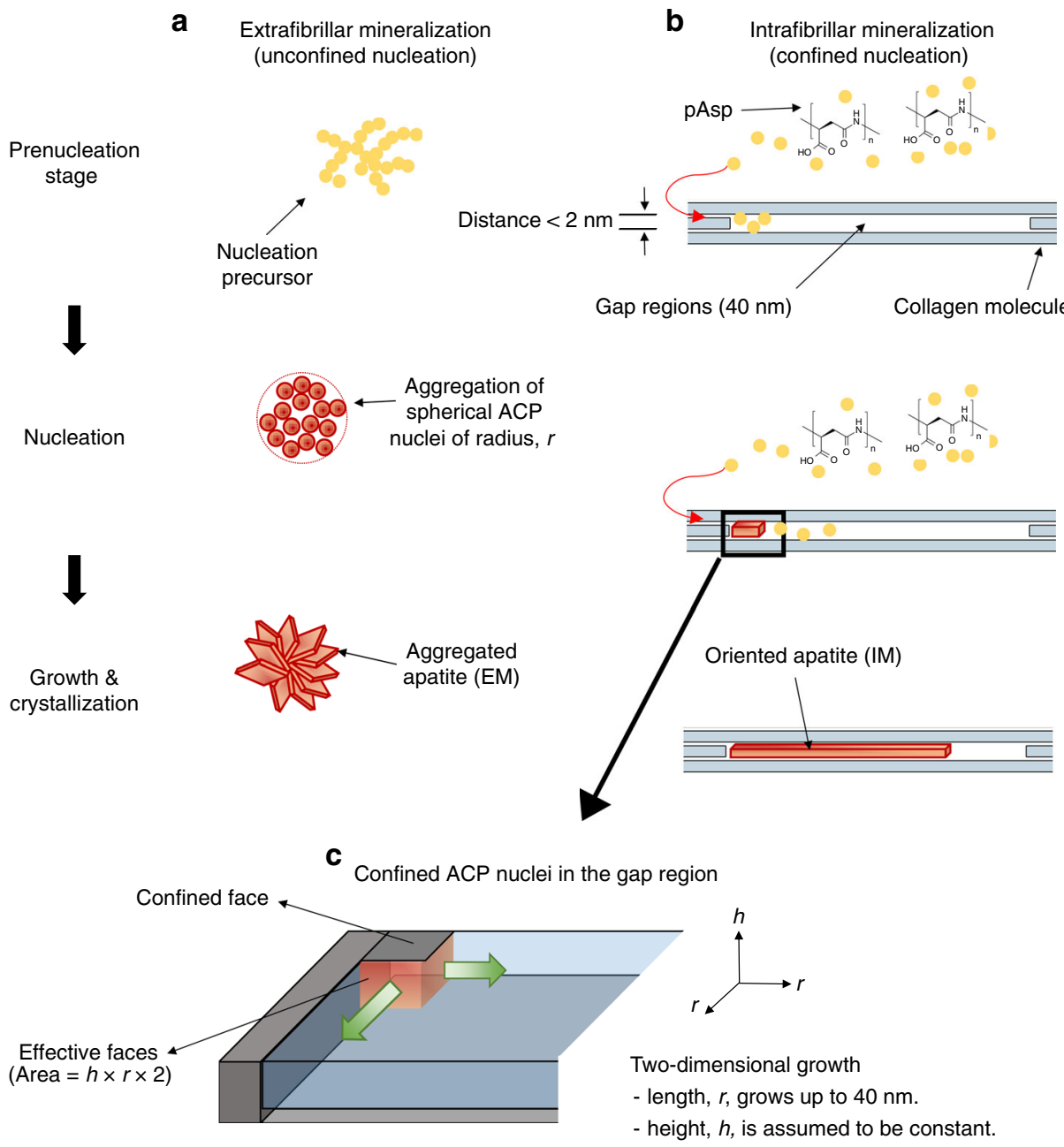

Fig. 1 Schematic illustration of two different nucleation models for collagen mineralization. $\mathbf{a}$ Extrafibrillar nucleation in unconfined space and $\mathbf{b}$ intrafibrillar nucleation in a confined gap region. c Geometry of confined amorphous calcium phosphate (ACP) nuclei in the gap region 
which was observed only during $\mathrm{EM}^{23}$, reduces the energy barrier to ACP nucleation ${ }^{25}$, while the intermediate stage did not appear during IM. Therefore, a higher nucleation barrier is expected for IM than EM. However, in most in vitro collagen mineralization studies, IM is more favored than EM in the presence of nucleation inhibitors ${ }^{20,23,27}$. Thus far, no study has separately evaluated the nucleation energy barriers for the two mineralization patterns to the best of our knowledge, so it is unclear how the confined collagen gap region contributes to overcoming the high nucleation energy barrier to IM.

Here, we present experiments using in situ small-angle X-ray scattering (SAXS) analysis to examine $\mathrm{CaP}$ nucleation rates during EM and IM in simulated body fluids (SBF) with or without pAsp (Supplementary Table 1 and Fig. 1). These data allow us to evaluate the nucleation energy barriers for IM and EM separately by applying the principles of classical nucleation theory $(\mathrm{CNT})^{28-31}$. To better determine nucleation patterns in the confined gap region, a new model was developed with an assumption of plate-like nuclei formation. Based on the CNT analysis, EM occurs initially in SBF solutions, due to their lowest nucleation energy barrier. The addition of pAsp kinetically inhibits EM formation; instead, it leads nucleation to occur dominantly in the narrow collagen gap regions, despite the increased interfacial energy there. In these highly confined spaces, nuclei grow in two-dimensions (2D), limiting the reactive surface area for nucleation and decreasing the surface energy contribution to the barrier. With this observation, we provide an explanation for how the highly confined spaces of the fibrillar collagen structure foster biomineralization.

\section{Results}

CNT application to extra and intrafibrillar mineralization. CNT has often been adopted to evaluate the interfacial energy between nuclei and a mineralization solution, and to evaluate the nucleation energy barrier ${ }^{28-31}$. CNT has provided fundamental knowledge about the nucleation and growth of biominerals, and recent studies have expanded its scope to interpret non-classical nucleation behaviors ${ }^{25,32-34}$. A heterogeneous nucleation model in CNT has also been adopted to explain how the interfacial energy and nucleation barrier decrease in the presence of collagen, by assuming the collagen is a flat substrate for hemispherical particle formation ${ }^{25,35-37}$. However, without a proper evaluation of the differences between EM and IM, the role of collagen in nucleation remains elusive. In addition, a careful consideration of collagen geometry is needed to properly account for the influence of the confinement of nuclei in the gap regions on IM.

According to CNT, nucleation through monomer-bymonomer attachment is assumed to be thermodynamically driven by the free energy change per molecule, $\Delta G$. For the formation of a nucleus from a solution, $\Delta G$ is given by the sum of the bulk $\left(\Delta G_{\mathrm{b}}\right)$ and surface $\left(\Delta G_{\mathrm{s}}\right)$ terms. A typical $\Delta G$ profile shows a maximum (i.e., an energy barrier, $\Delta G_{n}$ ) at a critical radius $\left(r_{c}\right)$, then decreases with increasing radius $(r)$. The nucleation rate $(J)$ can be expressed with $\Delta G_{\mathrm{n}}$ in its exponential term, $J=A \exp \left(-\frac{\Delta G_{n}}{k_{B} T}\right)$, where $k_{\mathrm{B}}$ is the Boltzmann constant, $T$ is the Kelvin temperature, and $A$ is a kinetic factor. For the formation of a spherical nucleus, $\ln (J)$ shows a linear relationship with $1 / \sigma^{2}$. Here the supersaturation $(\sigma)$ of the solution is given as $\ln (\mathrm{IAP} /$ $K_{\mathrm{sp}}$ ), where IAP is the ion activity product and $K_{\mathrm{sp}}$ is the solubility product. In this linear relationship, the fitting of the slope $(B)$, as listed in Table 1, can provide the interfacial energy $(\alpha)$ between nuclei and the mineralization solution. We applied this relationship to evaluate $\alpha$ for EM, where nucleation occurs in relatively unconfined macroscale spaces, with the assumption of spherical nuclei formation (Fig. 1a). This assumption is reasonable because the EM pathway forms spherical ACP nuclei ${ }^{23-25}$.

On the other hand, we previously found that plate-like $\mathrm{CaP}$ formed in the confined gap region without intermediate spherical $\mathrm{ACP}^{23}$. Similar 2D crystallization can occur by the preferential adsorption of small acidic molecules, such as phosphoserine and citrate, on specific faces of apatite nuclei ${ }^{38,39}$. To quantitatively evaluate nucleation in the confined gap region (IM), we developed a model of nucleation in which $2 \mathrm{D}$ crystals grow only in the lateral directions with a uniform height (Fig. 1b). The relevant equations are given in Table 1. A plate-like morphology was assumed to better reflect the CNT precept that the nucleus and final crystal have the same crystalline structure. In this model, the surfaces of nuclei confined in the collagen gap region do not allow monomer-by-monomer attachments of precursor molecules for nucleation. Only the exposed surfaces can affect the

Table 1 Derivation of the nucleation energy barrier $\left(\Delta G_{n}\right)$ and interfacial energy $(\alpha)$ of the unconfined nucleation model for extrafibrillar mineralization (EM) and the confined nucleation model for intrafibrillar mineralization in the collagen gap region (IM), based on classical nucleation theory

Extrafibrillar mineralization (Unconfined nucleation) Intrafibrillar mineralization (Confined nucleation)

$$
\begin{aligned}
& \hline \text { Morphology of nucleus } \\
& \text { Effective surface area exp } \\
& \text { Volume } \\
& \Delta G=\Delta G_{\mathrm{b}}+\Delta G_{\mathrm{s}} \\
& r_{\mathrm{c}}(\text { at } \mathrm{d} \Delta \mathrm{G} / \mathrm{d} r=0) \\
& \Delta G_{\mathrm{n}}\left(\text { at } r=r_{\mathrm{c}}\right) \\
& J=\mathrm{A} \exp \left(-\Delta G_{\mathrm{n}} / k_{\mathrm{B}} T\right) \\
& \ln (J) \\
& \alpha
\end{aligned}
$$

\section{Sphere}

Effective surface area exposed to solution $4 \pi r^{2}$$$
\frac{2 v_{\mathrm{m}} \alpha}{k_{\mathrm{B}} \sigma \sigma}
$$$$
\text { B }
$$$$
\frac{16 \pi v_{\mathrm{m}}^{2} \alpha^{3}}{3 k_{\mathrm{B}}^{2} T^{2} \sigma^{2}}
$$

$$
\begin{aligned}
& \text { Plate (constant } h \text { ) } \\
& 2 r h \text { (two edge surfaces) } \\
& r^{2} h \\
& -\left\{\frac{\left[r^{2} h\right]}{v_{m}}\right\} k_{B} T \sigma+2 r h \alpha \\
& \frac{v_{m} \alpha}{k_{B} T \sigma} \\
& \frac{h v_{m} \alpha^{2}}{k_{B} T \sigma} \\
& A \exp \left(-\frac{h v_{m} \alpha^{2}}{k_{B}^{2} T^{2} \sigma}\right) \\
& \ln (A)-B \frac{1}{\sigma^{\prime}} B=\left(\frac{h v_{m} \alpha^{2}}{k_{B}^{2} T^{2}}\right) \\
& \left(\frac{B k_{B}^{2} T^{2}}{h v_{m}}\right)^{\frac{1}{2}}
\end{aligned}
$$$$
-\left\{\frac{\left[\left(\frac{4}{3}\right) \pi r^{3}\right]}{v_{\mathrm{m}}}\right\} k_{\mathrm{B}} T \sigma+4 \pi r^{2} \alpha
$$$$
A \exp \left(-\frac{16 \pi v_{m}^{2} \alpha^{3}}{3 k_{B}^{3} T^{3} \sigma^{2}}\right)
$$$$
\ln (A)-B \frac{1}{\sigma^{2}}, B=\left(\frac{16 \pi v_{\mathrm{m}}^{2} \alpha^{3}}{3 k_{\mathrm{B}}^{3} T^{3}}\right)
$$$$
\left(\frac{3 B k_{B}^{3} T^{3}}{16 \pi v_{m}^{2}}\right)^{\frac{1}{3}}
$$

The effect of confinement on the shape and growth of nuclei for the confined nucleation model is illustrated in Fig. 1. The morphology of the nucleus changes the parameters in the bulk and surface energy terms $\left(\Delta G_{\mathrm{b}}\right.$ and $\Delta G_{\mathrm{s}}$, respectively). As a result, $\ln (J)$ shows a linear relationship with $1 / \sigma^{2}$ for unconfined nucleation, but with $1 / \sigma$ for fully confined nucleation

$r$ and $h$ are the radius (or length for the confined nucleation model) and height of nuclei. For the volume per molecule of nucleus, $v_{\mathrm{m}}, 5 \times 10^{-23} \mathrm{~cm}^{3}$ and $2.63 \times 10^{-22} \mathrm{~cm}^{3}$ are used for ACP25 and for $\mathrm{HA}^{66}$ respectively. $k_{\mathrm{B}}$ is the Boltzmann constant $\left(1.38 \times 10^{-23} \mathrm{~J} \mathrm{~K} \mathrm{~K}^{-1}\right) . T$ is the temperature of the reactor $(310 \mathrm{~K}) . \sigma$ is the supersaturation (In $\left.\left(\mathrm{IAP} / \mathrm{K}_{\mathrm{sp}}\right)\right)$, where IAP is the ion activity product and $K_{\mathrm{sp}}$ is the solubility product. $\alpha$ is the interfacial energy between nuclei and solution 
a

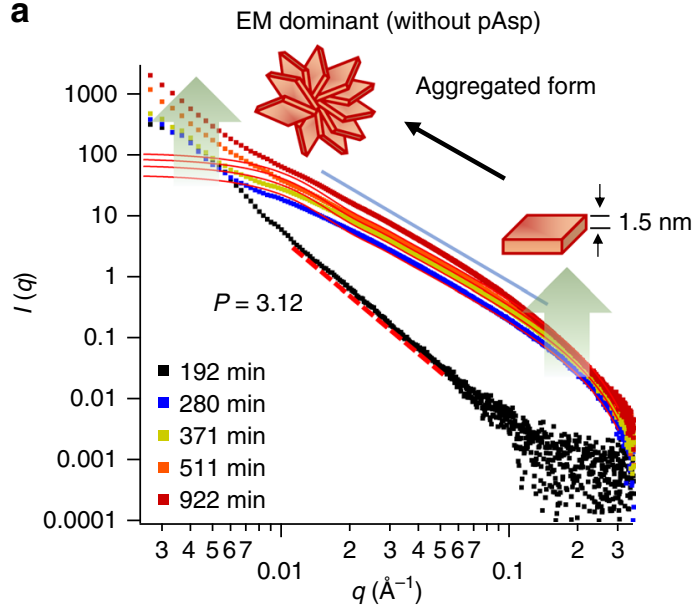

b

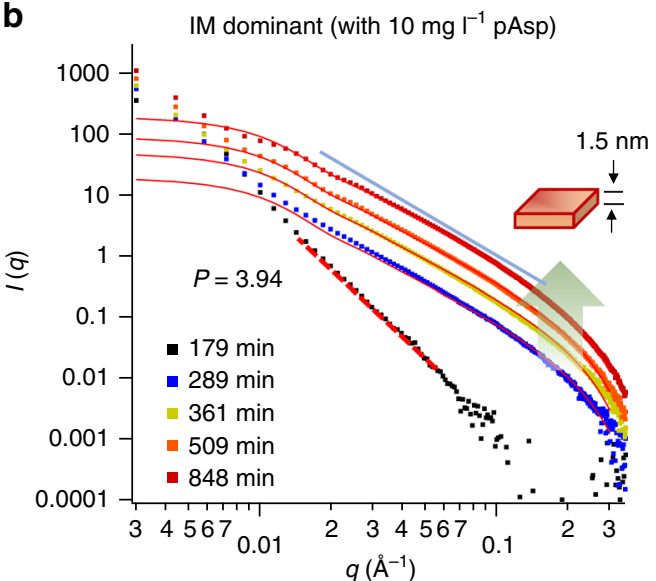

c

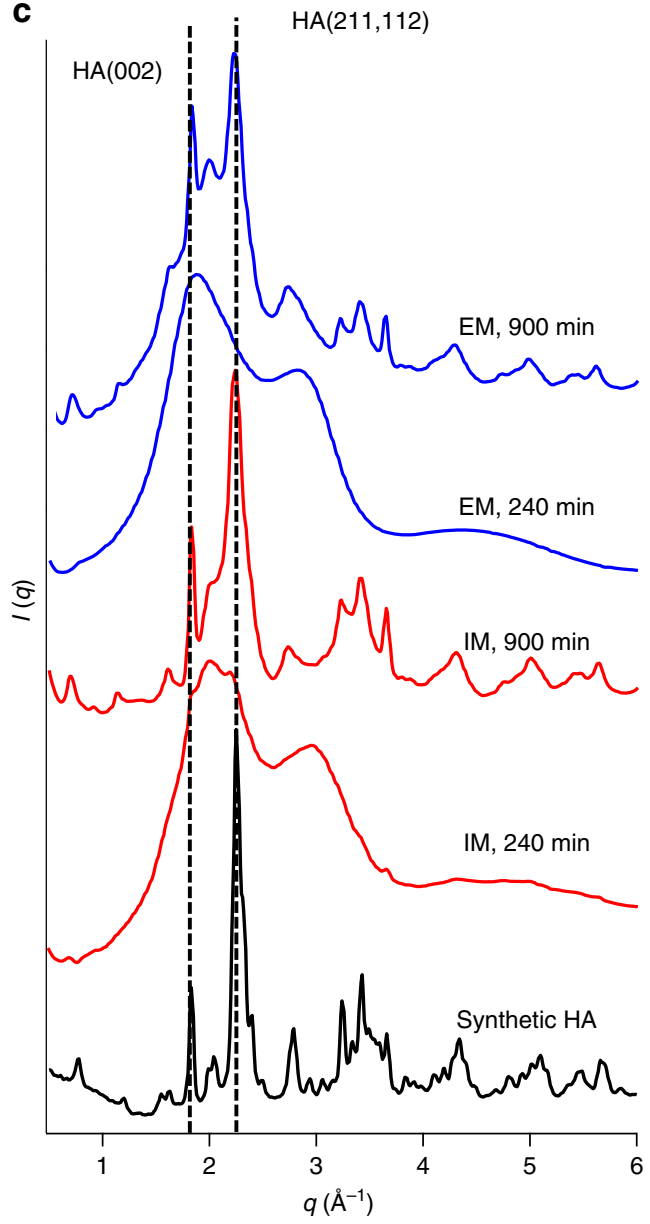

Fig. 2 In situ SAXS/WAXD patterns from collagen matrices during mineralization. Data collected from unmineralized collagen was used for background subtraction. a, b Small-angle X-ray scattering (SAXS) patterns collected during mineralization without pAsp for extrafibrillar mineralization (EM, a) and with pAsp for intrafibrillar mineralization (IM, b) in the $2.85 \times$ SBF solution. Red solid lines fit plate-like particles (height: $1.5 \mathrm{~nm}$ and length: $40 \mathrm{~nm}$ ). $P$ values are the slopes of the Porod regime at 179 and $192 \mathrm{~min}$, in red dotted lines $\left(I(q) \propto q^{-P}\right)$. The solid light blue lines show negative slopes of 2 in the $\log$ - log plot after the induction time, indicating the 2-dimensional morphology of nuclei. c Wide-angle X-ray diffraction (WAXD) patterns of CaP formed in collagen matrices at early $(240 \mathrm{~min})$ and later $(900 \mathrm{~min})$ stages of mineralization. Synthetic hydroxyapatite (HA) was analyzed for comparison

calculation of $\Delta G_{s}$ (Fig. 1c) ${ }^{40}$. To simulate the reported IM nucleation initiated at the specific band positions near C-terminal ends of the gap region ${ }^{14,20,41}$, we assumed that nuclei formed at a corner of the collagen gap region (two edge surface exposure). The nuclei then thermodynamically benefit by minimizing their surface area to form a solid phase in an aqueous solution. In this model, $\ln (J)$ shows a linear relationship with $1 / \sigma$, which also provides $\alpha$ (between the edge surfaces of nuclei and the solution) from the fitting of the slope $B$ (Table 1$)$. When the confinement effect is considered, the different assumptions about the morphology and effective surfaces of nuclei do not change this linear relationship and consequent $\Delta G_{\mathrm{n}}$ values (Supplementary Note 1). Therefore, in this study, we mainly evaluated the comparison between confined and unconfined models, instead of exploring other possible scenarios of nucleation occurring in this confined space.

Extra and intrafibrillar mineralization controlled by pAsp. To evaluate the differences in $\alpha$ and $\Delta G_{\mathrm{n}}$ between IM and EM, nucleation rates must be separately measured. The two mineralization behaviors were controlled using pAsp. Our previous study also utilized pAsp in SBF solution with three times the usual concentrations of $\mathrm{Ca}$ and $\mathrm{P}(3 \times \mathrm{SBF})$ and showed how it controlled these two mineralization behaviors ${ }^{23}$. In the current study, scanning electron microscopy (SEM) images revealed that the addition of $10 \mathrm{mg} \mathrm{l}^{-1}$ pAsp successfully separated EM and IM patterns in a wide range of $\sigma$, using $2.65-3.0 \times$ SBF solutions (Supplementary Note 2).

The differences in nucleation behaviors between EM and IM controlled by pAsp were also apparent in SAXS patterns (Fig. 2a, b). For example, SAXS intensities in the small $q$ region $\left(<0.01 \AA^{-1}\right)$ continuously increased during EM without pAsp over time. The intensity increases in the small $q$ region were mainly caused by the formation of particles larger than $62.8 \mathrm{~nm}$ in diameter $(d=$ $2 \pi / q)$. Without pAsp, such large particles were observed only as an aggregated form of $\mathrm{CaP}$ in the extrafibrillar space (Supplementary Fig. $3 \mathrm{c}, \mathrm{d})$. The SAXS patterns in the large $q$ region $\left(>0.01 \AA^{-1}\right.$, Fig. $2 \mathrm{a}$ at after $\left.280 \mathrm{~min}\right)$ fit well with plate-like particles with a uniform height of $1.5 \mathrm{~nm}$. The negative slope of 2 between the two extreme regions, at $q$ around $0.01-0.2 \AA^{-1}$, is evidence of the $2 \mathrm{D}$ structure of a plate-like particle ${ }^{42-44}$. Thus, we concluded that these patterns represent aggregates of thin apatite crystals, as commonly observed during EM (Supplementary Fig. 3d). On the other hand, during IM with $10 \mathrm{mg}^{-1} \mathrm{pAsp}$, similar plate-like particles ( $1.5 \mathrm{~nm}$ height) developed without forming an aggregate (little increases of SAXS intensities at small $q$, Fig. 2b). This observation indicates that individual plates are separately arranged within the collagen, as expected from IM. 
Evidence of aggregation during EM can also be found from the negative slope, $P$, of the Porod regime, $q>0.2 \AA^{-1}$, at the induction period before plate-like particle development. During EM without pAsp, a $P$-value close to 3 (at $192 \mathrm{~min}$, Fig. 2a) indicates an isometric mass fractal structure, such as a spherical aggregate. This slope is clearly distinct from that of a typical compact object $(P=4)$, such as appeared during the induction period of IM (at $179 \mathrm{~min}$, Fig. $2 \mathrm{~b})^{23,42}$.

Wide-angle X-ray diffraction (WAXD) of samples indicated that very little crystalline structure developed, either because the structure was mostly amorphous or because the nuclei were few and small, at the beginning of both intrafibrillar and extrafibrillar mineralization (Fig. 2c, <240 min). However, these patterns developed to HA-like, yet poorly crystalline, crystals at a later stage ( $>900 \mathrm{~min}$ ). In the presence of pAsp (IM case), from the beginning, a slightly higher crystallinity developed from nucleated minerals. In other words, with pAsp, enhanced intensities were observed at $q=1.83,2.23$, and $2.26 \AA^{-1}$ (corresponding to (002), (211) and (112) reflections, respectively), while only a smooth hump appeared around $q=1.83 \AA^{-1}$ without pAsp (EM case). These data show that the crystallinity of nuclei developed more slowly during EM than IM, even though the total particle volume in this period, as quantified from the absolute SAXS intensity ${ }^{45}$, was slightly higher for EM. EM essentially undergoes a phase transformation pathway involving an amorphous intermediate product, delaying the transformation to HA-like crystals ${ }^{23}$.

Because amorphous phases were dominant in WAXD patterns at the early stage of mineralization, we assumed that nuclei were amorphous in further studies exploring the interfacial energy relationship. Many previous studies have also hypothesized ACP as a precursor phase of bioapatite in bones ${ }^{20,25,46-48}$. However, there remains a possibility of direct formation of crystalline apatite during IM, because a sudden formation of plate-like particles was observed in our recent report ${ }^{23}$. Indeed, the absence of ACP has been reported in studies of young bones ${ }^{49}$ and biomimetic nanocrystalline $\mathrm{HA}^{50}$. Therefore, we also provided the relationship based on the assumption of HA nuclei, which is comparable to previous reports examining the interfacial energy for HA nucleation ${ }^{25,35,37}$.

Nucleation rates measured by in situ SAXS analysis. SAXS patterns collected at the nucleation stage suggest the formation of plate-like particles for both EM and IM. The invariant value, $Q=\frac{1}{2 \pi^{2}} \int q^{2} I(q) \mathrm{d} q$, is a quantity proportional to the total particle volume ${ }^{51}$. Therefore, $J$ was determined from the slope of $Q$ vs. time plots during mineralization in different SBF solutions (Fig. 3a, b), with the assumption of constant morphology and electron density of the nuclei. During EM development without pAsp (Fig. 3a), $Q$ values increased after 160-290 min of induction (x-intercepts), then reached a plateau around $Q=4 \times 10^{-5}$ for all $\mathrm{SBF}$ solutions. At the SBF/collagen matrix interface, a thin layer of calcium phosphate crystals formed, acting as a diffusion barrier to the molecules required for the mineralization of the inner side of the matrix ${ }^{23}$. Therefore, only the outer surfaces of the collagen matrices could serve as nucleation sites. On the other hand, during IM with pAsp, the milder slopes of $Q$ and longer induction times (230-500 min) than for EM indicate that nucleation was inhibited by $\mathrm{pAsp}$ at the early stage ${ }^{23}$. However, the $Q$ values pass through the maximum plateau value obtained from EM, showing continuous and linear increases over $900 \mathrm{~min}$ (Fig. 3b). Because no diffusion barrier formed during IM, the entire volume of the collagen matrices could be mineralized.

Interfacial energies and energy barriers for EM and IM. Separately obtained $J$ values for EM and IM were applied to our
CNT models for EM $\left(\ln (J) \propto 1 / \sigma^{2}\right.$, Fig. 3c) and $\operatorname{IM}(\ln (J) \propto 1 / \sigma$, Fig. 3d). In this way, we could quantify the interfacial energies between $\mathrm{CaP}$ nuclei and SBF solutions in unconfined extrafibrillar space and confined gap regions. The $\alpha$ value for IM was calculated to be about four times higher than that for EM with respect to $\operatorname{ACP}\left(\alpha_{\mathrm{ACP}}=19 \pm 1 \mathrm{~mJ} \mathrm{~m}^{-2}\right.$ for IM vs. $5 \pm 1 \mathrm{~mJ} \mathrm{~m}^{-2}$ for EM). Due to this significant difference in $\alpha$, a small concentration of pAsp could effectively control the nucleation behavior, while not significantly decreasing the $\sigma$ of the bulk SBF solutions. The amount of pAsp used $\left(10 \mathrm{mgl}^{-1}\right)$ was equivalent to only 0.072 $\mathrm{mM}$ of aspartyl residue $\mathrm{e}^{52}$, therefore the change in $\sigma$ by complexation between ionic calcium species and pAsp was $<5 \%$ (Supplementary Table 1). Due to the increased $\alpha, \Delta G_{\mathrm{n}}$ for ACP nucleation is typically higher for IM than for EM over a wide range of $\sigma$ (Table 1 and Fig. $4 \mathrm{a}$, red and blue lines), including our experimental range $\left(\sigma_{\mathrm{ACP}}=0.60-1.13\right.$, Supplementary Table 1$)$. The ratio of the $\Delta G_{\mathrm{n}}$ for IM over the $\Delta G_{\mathrm{n}}$ for EM, however, decreases with decreasing supersaturation $\left(\Delta G_{\mathrm{n}, \mathrm{IM}} / \Delta G_{\mathrm{n}, \mathrm{EM}}=\right.$ $\left.\frac{3 h k_{\mathrm{B}} T \alpha_{\mathrm{IM}}^{2}}{16 \pi v_{\mathrm{m}} \alpha_{\mathrm{EM}}^{3}} \sigma=18.8 \sigma\right)$. Eventually, at $\sigma_{\mathrm{ACP}}<0.05, \Delta G_{\mathrm{n}, \mathrm{IM}}$ becomes lower than $\Delta G_{\mathrm{n}, \mathrm{EM}}$ (Fig. $4 \mathrm{~b}$ ), making IM more favorable than EM in this $\sigma$ range. The low supersaturation degree of the body fluid with respect to ACP, therefore, could be an important factor guiding nucleation in the confined gap regions.

Although human blood plasma is highly supersaturated with respect to $\mathrm{HA}$, our thermodynamic calculation shows that it is undersaturated with ACP ( $\sigma<0$, Supplementary Table 1$)$. However, it has been suggested that the concentrations of ionic components, such as $\mathrm{Ca}^{2+}$ and $\mathrm{HPO}_{4}{ }^{2-}$, can increase during fibrillogenesis of the collagen scaffold ${ }^{10}$. The extracellular $\mathrm{pH}$ might be higher near the bone-forming zone than in body fluids, because bone serves as a massive reservoir of alkaline materials ${ }^{53}$. Therefore, body fluids might be locally saturated to form ACP. Indeed, aggregated ACP particles were more abundant on the surface of newly forming bones than in other areas in the extracellular space of embryonic chickens ${ }^{54}$. Thus, we expect the actual $\sigma$ value with respect to ACP near bone-forming zones is slightly higher than zero.

When the plate-like CaP nucleus is confined to finite thickness, $h$, its edge surfaces can have a convex curvature, $r=\frac{h}{2}$. The solubility increase of the confined $\mathrm{CaP}$ mineral compared to its bulk property can be calculated using the modified Kelvin equation, $\frac{S_{r}}{S_{0}}=\exp \left(\frac{2 \alpha V}{R T r}\right)^{55}$, where $S_{\mathrm{r}}$ and $S_{0}$ are the solubility in confined space and the solubility of bulk, respectively. $V$ is the molecular volume in $\mathrm{m}^{3} \mathrm{~mol}^{-1}$, and $R$ is the gas constant. With a strong wettability of the nucleus or chemical affinity toward the confining walls, the nucleus would have concave edges ${ }^{56,57}$. Then, the Kelvin equation reads $\frac{S_{r}}{S_{0}}=\exp \left(-\frac{2 \alpha V}{R T r}\right)$ for negative curvature $-r$. According to the Kelvin equation, the confinement increases solubility by $3 \%$ with a convex curvature and decreases it by $2 \%$ with a concave curvature (Supplementary Fig. 4). The decreased solubility of $\mathrm{CaP}$ with a concave edge contributes to reducing $\Delta G_{\mathrm{n}}$ and facilitating 2D crystallization in the confined space (Supplementary Fig. 5). For convex edges, the contribution of increased solubility to $\Delta G_{\mathrm{n}}$ is not significant, and therefore, confined nucleation is still preferred over nucleation without confinement.

By evaluating an IM model with no confined effect, we clearly demonstrate that the confined collagen structure contributes further to reducing the nucleation energy barrier. We assumed that nucleation occurs in the presence of pAsp (using $\alpha_{\mathrm{ACP}}$ for IM), but without consideration of the confined collagen geometry (unconfined model). $\Delta G_{\mathrm{n}, \mathrm{IM}}$ was smaller than $\Delta G_{\mathrm{n}}$ for the unconfined IM model ( $\left.\Delta G_{\mathrm{n} \text {,unconf }}\right)$ over the entire $\sigma_{\mathrm{ACP}}$ range, and the difference became larger at lower $\sigma\left(\Delta G_{\mathrm{n}, \text { unconf }} / \Delta G_{\mathrm{n}, \mathrm{IM}}=\right.$ 

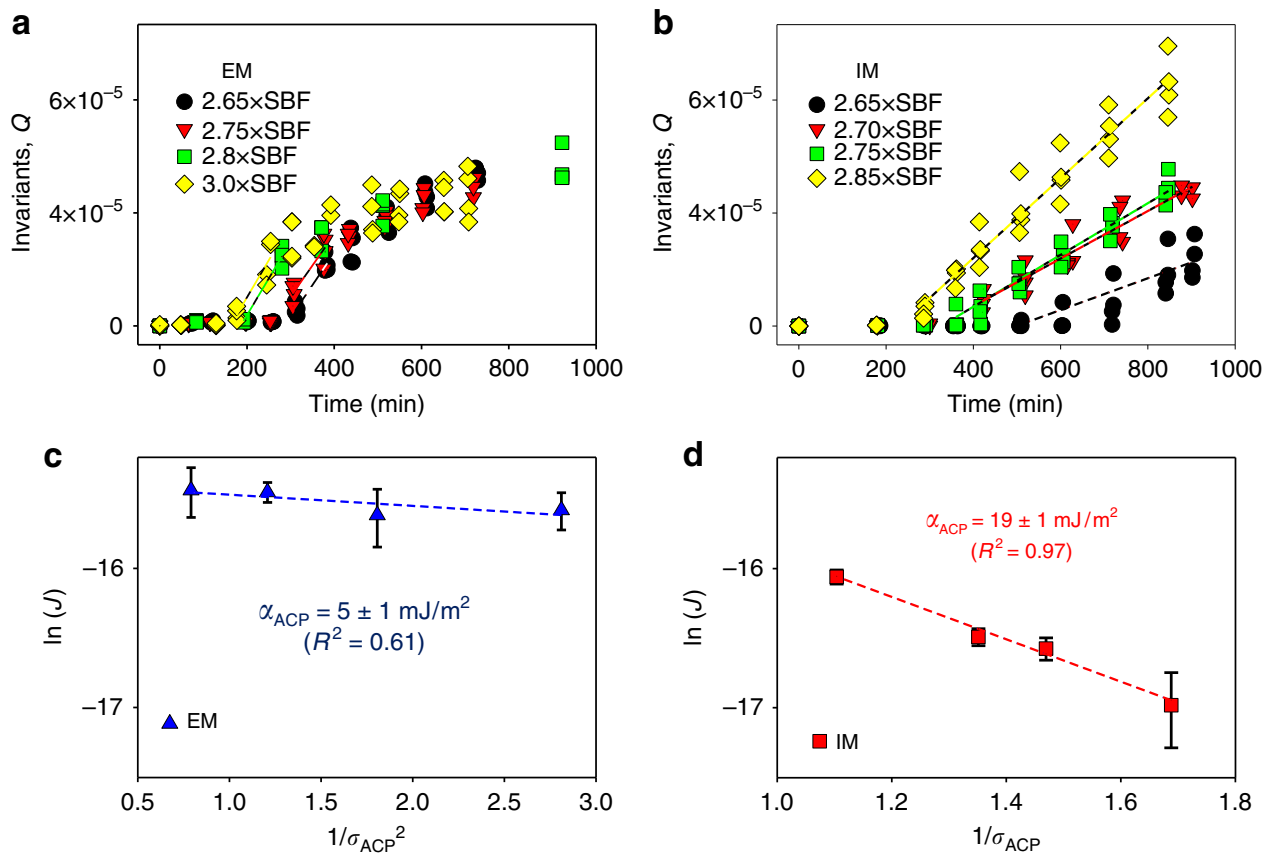

Fig. 3 Interfacial energy relationships during the nucleation of calcium phosphate within collagen fibrils. $\mathbf{a}, \mathbf{b}$ Evolution of the invariant, Q, from in situ SAXS measurements of collagen matrices in different simulated body fluid (SBF) solutions without pAsp (a, representing extrafibrillar mineralization, EM) and with pAsp (b, representing intrafibrillar mineralization, IM). The slopes of the dotted lines indicate the nucleation rate, J. For the EM case, the initial $J$ values were taken from the maximum slopes between two time intervals. Only $Q$ within the range of $q=0.05-0.3 \AA^{-1}$ (corresponding to plate-like particles) were calculated. c, $\mathbf{d}$ Interfacial energies for ACP nucleation $\left(\alpha_{\mathrm{ACP}}\right)$ during EM and IM, calculated from the relationship between $J$ and supersaturation with respect to $A C P, \sigma_{A C P}$ (see Table 1 for the equations). Error bars in the symbols indicating $\ln (J)$ are standard errors of the estimates, obtained from the regressions between $Q$ and time (from $\mathbf{a}, \mathbf{b}$ ). Error ranges for $\alpha_{\mathrm{ACP}}$ values for EM and IM are standard errors of the estimates for regressions between In $(J)$ and $1 / \sigma^{2}$, and between $\ln (J)$ and $1 / \sigma$, respectively

$\frac{16 \pi v_{\mathrm{m}} \alpha_{\mathrm{IM}}}{3 h k_{\mathrm{B}} T \sigma}=2.5 / \sigma$, Fig. 4a). We also evaluated $\alpha$ values based on the $\mathrm{HA}$ nuclei assumption $\left(\alpha_{\mathrm{HA}}=267 \pm 27\right.$ (IM) vs. $58 \pm 8$ (EM) mJ $\mathrm{m}^{-2}$, Supplementary Fig. 6). $\Delta G$ profiles for the three cases (EM, IM, and unconfined IM) are shown in Supplementary Fig. 7.

\section{Discussion}

In previous studies, collagen has been examined as a substrate promoting the formation of calcium phosphate crystals via heterogeneous nucleation $24,25,30$. However, the promoted nucleation by collagen is somewhat contradictory to other studies, which suggest that a nucleation inhibitor is required for in vitro mineralization in gap regions ${ }^{20,23}$. Our evaluation of $\Delta G_{n}$ for three different scenarios provides a thermodynamic explanation for how the nucleation inhibitor and confined collagen gap combine to drive IM. Without pAsp, EM is the preferred pathway for $\mathrm{CaP}$ nucleation over a wide $\sigma$ range. pAsp is a strong regulator, preventing undesirable nucleation in the extrafibrillar space by increasing $\alpha$. In an environment where pAsp (or other nucleation inhibitors) are present, nuclei seek nucleation sites with a lower energy barrier. The confined collagen gap region provides such sites for nuclei because the space effectively reduces $\Delta G_{\mathrm{s}}$ by minimizing the effective surface area of nuclei.

By separately evaluating the $\alpha$ values for EM and IM, for the first time, we report that this value varies significantly depending on the nucleation site. The $\alpha_{\mathrm{HA}}$ values provided by previous studies $\left(90 \mathrm{~mJ} \mathrm{~m}^{-2}\right.$ by Habraken et al. ${ }^{25}$ and $105 \mathrm{~mJ} \mathrm{~m}^{-2}$ by Koutsoukos and Nancollas ${ }^{35}$, with no distinction between EM and IM) were approximately in the middle of the range of values that we obtained (58 and $267 \mathrm{~mJ} \mathrm{~m}^{-2}$ for EM and IM). Thus, the current approach can be used to identify the contributions of EM and IM in different biomimetic environments, providing insights into biomaterials with multi-scale pore structures.

The findings could also help identify important mechanisms governing biomineralization, although with significant uncertainties due to the complexity of physiologic systems. The uncertainty is exaggerated for ACP, whose physicochemical properties remain unclear. The $\alpha_{\mathrm{ACP}}$ values evaluated in this study are lower (5 and $19 \mathrm{~mJ} \mathrm{~m}^{-2}$ for EM and IM) than in the previous study by Habraken et al. ${ }^{25}\left(40 \mathrm{~mJ} \mathrm{~m}^{-2}\right.$ for heterogeneous nucleation on a collagen-coated substrate, assuming hemispherical nuclei). Their study used in situ atomic force microscopy (AFM) to determine nucleation rates, which could not distinguish between IM and EM, and thus did not account for the confinement in collagen structures. Because AFM is a surface technique, this study might probe mainly the EM on the surface. The complexities of the ionic compounds found in SBF solutions (which would notably be even more complex in physiologic systems) can also influence $\alpha$. Ionic components, such as $\mathrm{Mg}^{2+}$ and $\mathrm{HCO}_{3}{ }^{-}$, can make SBF solutions more favorable to forming $\mathrm{ACP}$, although they do not change the $\sigma$ of the solutions significantly ${ }^{58}$.

To better evaluate the complexity of the physiological system, the roles of NCPs in collagen mineralization should be also carefully examined in future studies. For example, mineralization of collagen may be decreased when NCPs are removed ${ }^{59}$. A recent immunocytochemistry study showed that osteocalcin was present in both gap and overlap regions, while bone sialoprotein was located only at the surface of or outside type I collagen of gastrocnemius tendons extracted from turkeys ${ }^{21}$. Therefore, the type(s) of NCPs and their spatial distributions within or near collagen fibrils may influence calcium phosphate deposition by altering the sequence of amino acid side changes at the nucleation 

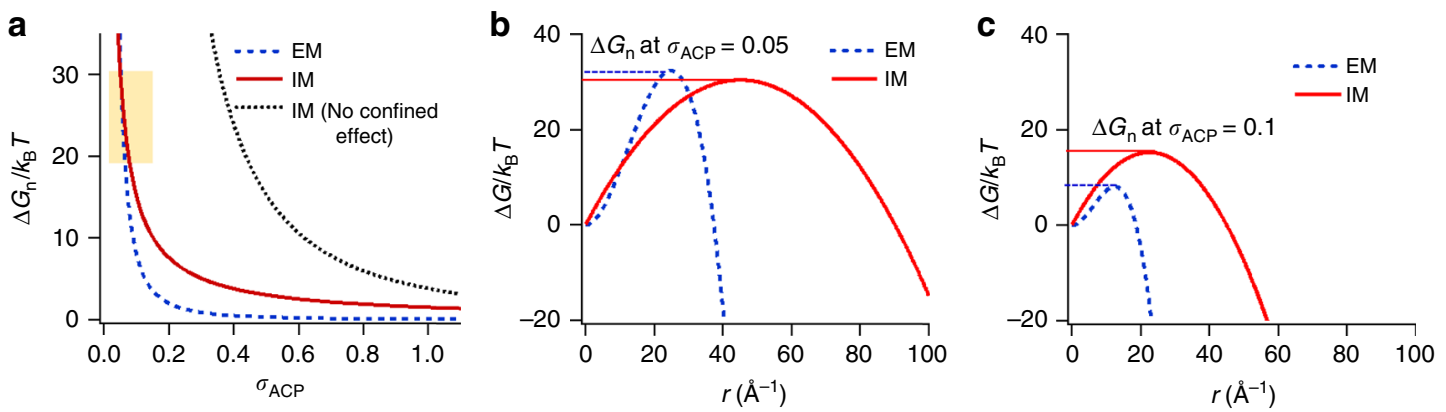

Fig. 4 Energy barriers to $A C P$ nucleation at different $\sigma_{A C P}$. a $\Delta G_{n}$ for three different nucleation models: unconfined nucleation without pAsp (representing extrafibrillar mineralization, EM), confined nucleation with pAsp (representing intrafibrillar mineralization, IM), and unconfined nucleation with pAsp (IM with no confined effect). b, c $\Delta G$ profiles at $\sigma_{\mathrm{ACP}}=0.05$ and 0.1 (yellow box in Fig. 4a)

sites $^{41}$. A better understanding of the NCP distributions would provide important insight into how organisms control mineral deposition to meet the specific requirements of mineralized tissues while using the same template, type I collagen. In the current study, we used an idealized model system to simulate one specific role of NCPs. pAsp was added to prevent nucleation of mineral in the bulk solution and allow nucleation of mineral in the confined collagen fibril spaces. This approach allowed us to determine the interfacial energy relationship of the IM-dominant system without the complications that would arise from the use of NCPs, which may have multiple functions during mineralization.

In this study, we evaluate $\mathrm{CaP}$ nucleation in the collagen gap regions where the equilibrium $\mathrm{CaP}$ solubility is influenced by the confinement. The confinement can also affect other physicochemical properties of $\mathrm{CaP}$ and fluid. Based on the literature, these potential differences in the materials' properties in the confined spaces are expected to make the energy barrier for IM even smaller than we estimated ${ }^{5,25,41,60}$. For example, the value of $\alpha$ might be particle size-dependent, and the height of plate-like nuclei is more likely in a range where $\alpha$ is decreasing from the value at its bulk phase ${ }^{25,60}$. Therefore, a smaller $\Delta G_{\mathrm{n}}$ is expected if the particle size-dependency is properly considered. It is known that decreasing particle size influences $\alpha$ through two opposing factors: The presence of high-energy sites can increase $\alpha$, but the structural similarity of the surface and interiors of nanoparticles may decrease it ${ }^{60}$. Although not experimentally proven yet for bioapatite, Habraken et al. suggested that a decreasing pattern of $\alpha$ would be valid for nuclei smaller than $3 \mathrm{~nm}$ in radius 25 . In our study, the height of plate-like nuclei measured by SAXS was 1.5 $\mathrm{nm}$, and the critical nucleus size for HA nuclei was calculated as less than $1 \mathrm{~nm}$ at $\sigma_{\mathrm{HA}}=22.7$ (body plasma condition, Supplementary Fig. 7). Another recent study calculated that the water density in the collagen gap regions was only about $0.7 \mathrm{~g} \mathrm{~cm}^{-3}$, which would similarly benefit $\mathrm{CaP}$ nucleation in this region by reducing the enthalpic penalty for ion desolvation ${ }^{41}$.

By combining in situ SAXS measurement and thermodynamic evaluation using $\mathrm{CNT}$, this study clearly shows that collagen fibrils provide nucleation sites for IM with a reduced nucleation energy barrier. These findings provide insight into bone mineralization occurring in the complex fibrillar collagen structure within a confined space and driven by extracellular proteins. Collagen fibrils were confirmed to play a significant role in biomineralization by controlling nucleation pathways and energy barriers; they do not therefore serve as passive templates.

\section{Methods}

Preparation of collagen matrices. Type I collagen (C857, calf skin lyophilized, Elastin Products Company, Inc.) was used to reconstitute collagen matrices ${ }^{23,61}$. Collagen was carefully dissolved in $0.5 \mathrm{mM} \mathrm{HCl}\left(12 \mathrm{mg} \mathrm{ml}^{-1}\right)$ at $4^{\circ} \mathrm{C}$ with magnetic stirring, followed by degassing under vacuum at $4^{\circ} \mathrm{C}$ for 4 days. The dissolved collagen solution was placed in two holes ( $3 \mathrm{~mm}$ in diameter) in a specially designed polytetrafluoroethylene frame with a thickness of $2.38 \mathrm{~mm}$ (Supplementary Fig. 1a). A \#1 cover glass was attached on one side of the frame to support the collagen solution during polymerization in TES buffered saline $(5.5,6.32$, and $3.4 \mathrm{gl}^{-1}$ of TES, $\mathrm{NaCl}$, and $\mathrm{Na}_{2} \mathrm{HPO}_{4}$, in deionized water, $\left.\mathrm{pH} 7.5\right)$ at $37 \pm 1^{\circ} \mathrm{C}$. The frame was then stored in deionized water overnight to remove excess salt. Based on our previous study ${ }^{23}$, the reconstituted collagen with a fibrillar density of $12 \mathrm{mg} \mathrm{ml}$ ${ }^{-1}$ was optimal for observing both intrafibrillar and extrafibrillar mineralization with SAXS. AFM imaging revealed $\sim 67 \mathrm{~nm}$ periodicity of the collagen, confirming that the nanoscale confinement distribution in the fibrils was comparable to native type I collagen (Supplementary Fig. 3a, b). In addition, the well-controlled shape and uniform thickness of the collagen matrix allowed for quantification of the nucleation rates using in situ SAXS at different time intervals.

Preparation of simulated body fluid solutions. Simulated body fluid (SBF) solutions were prepared using the method proposed by Kokubo et al. ${ }^{62}$ to mimic major ionic compounds in human body plasma ${ }^{63}$. American Chemical Society grades of $\mathrm{NaCl}$ (7.996 g, BDH Chemicals), $\mathrm{NaHCO}_{3}(0.350 \mathrm{~g}, \mathrm{BDH}$ Chemicals), $\mathrm{KCl}\left(0.224 \mathrm{~g}, \mathrm{BDH}\right.$ Chemicals), $\mathrm{MgCl}_{2} \cdot 6 \mathrm{H}_{2} \mathrm{O}(0.305 \mathrm{~g}$, EMD Millipore), $1 \mathrm{M} \mathrm{HCl}$ ( $40 \mathrm{ml}, \mathrm{BDH}$ Chemicals), $\mathrm{Na}_{2} \mathrm{SO}_{4}(0.071 \mathrm{~g}$, Alfa Aesar), and tris(hydroxymethyl) aminomethane (Tris, $6.057 \mathrm{~g}$, Alfa Aesar) were added to $900 \mathrm{ml}$ of deionized water (18.2 M $2-\mathrm{cm}$ ). Either 0 or $10 \mathrm{mg}$ of pAsp (sodium salt, Mw: 5,000 Da, LANXES) was added to the solution, depending on the experimental condition. Then the solution was equally separated into two $500 \mathrm{ml}$ polyethylene bottles, and either 0.604-0.684 g of $\mathrm{K}_{2} \mathrm{HPO}_{4} \cdot 3 \mathrm{H}_{2} \mathrm{O}$ (Alfa Aesar) or 0.974-1.103 g of CaCl $2 \cdot 2 \mathrm{H}_{2} \mathrm{O}$ (Alfa Aesar) was added to each bottle. The $\mathrm{pH}$ of the solutions was adjusted to 7.25 with $1 \mathrm{M} \mathrm{HCl}$, followed by filling up the volume with water to $500 \mathrm{ml}$. To prevent any precipitation of calcium phosphate minerals prior to the start of the experiment, the two stable solutions containing either $\mathrm{Ca}^{2+}$ or $\mathrm{HPO}_{4}{ }^{2-}$ precursors (SBF-Ca or SBF-P) were prepared separately and mixed just before the reaction (Supplementary Fig. 1b). The concentrations of $\mathrm{Ca}$ and $\mathrm{P}$ in the reactor, after the two solutions were mixed, were 2.65 to 3.0 times higher than in the SBF solution by Kokubo's method (2.65-3.0 $\times \mathrm{SBF})$, however, the $\mathrm{Ca} / \mathrm{P}$ molar ratio was constantly fixed to 2.5. The continuous flow-through reaction system (Supplementary Fig. 1b) allowed maintaining constant concentrations of ionic components and $\mathrm{pH}$ in the reactor for each set of experiment (details listed in Supplementary Table 1). The addition of $10 \mathrm{mgl}^{-1} \mathrm{pAsp}$, a nucleation inhibitor for EM, effectively promoted IM for up to $15 \mathrm{~h}$ during the mineralization of collagen matrices in the $3.0 \times \mathrm{SBF}^{23}$. The use of a buffer in the SBF solution was unavoidable in order to maintain the desired $\mathrm{pH}$. This buffer allowed us to use a constant supersaturation value for each SBF solution for the application of CNT. In this study, tris-buffer was used because it has been widely and effectively used for biomimetic CaP synthesis and intrafibrillar collagen mineralization ${ }^{23,58}$. More details about the potential influence of buffers in physiological solutions were summarized in a recent review paper ${ }^{58}$.

Supersaturation $(\sigma)$ values of the SBF solutions were calculated for both HA $\left(\sigma_{\mathrm{HA}}\right)$ and $\mathrm{ACP}\left(\sigma_{\mathrm{ACP}}\right)$. The ion activity product of hydroxyapatite $\left(\mathrm{IAP}_{\mathrm{HA}}\right)$ was defined as $\left(\alpha_{\mathrm{Ca}^{2+}}\right)^{5}\left(\alpha_{\mathrm{PO}_{4}^{3-}}\right)^{3}\left(\alpha_{\mathrm{OH}^{-}}\right)^{64}$. The activity of an ionic compound $i, \alpha_{i}$, is the product of its activity coefficient, $\gamma_{i}$, and concentration, $C_{i}$. We calculated $\gamma_{i}$ values at $37^{\circ} \mathrm{C}$ from the modified Debye-Hückel equation, $\log \gamma_{i}=-0.5211 z_{i}^{2}\left[\frac{\frac{1}{12}}{1+\frac{1}{12}}-0.3 I\right],{ }^{64,65}$, where $I$ is the total ionic strength $\left(I=\frac{1}{2} \sum C_{i} z_{i}\right)$ and $z_{i}$ is the charge number. To calculate $C_{i}$ of all the ionic components in SBF solutions, MINEQL + was used, with consideration of pAsp based on its dissociation and calcium binding constants as reported by Wu and Grant ${ }^{52}$. Using values from the literature ${ }^{66,67}$, we set $K_{\mathrm{sp}}$ (HA) $=2.35 \times 10^{-59}$ and set the molecular volume, $v_{\mathrm{m}}(\mathrm{HA})=2.63 \times 10^{-22} \mathrm{~cm}^{3} . \sigma_{\mathrm{ACP}}$ was calculated based on $\mathrm{Ca}_{2}\left(\mathrm{HPO}_{4}\right)_{3}{ }^{2-}\left(\mathrm{IAP}_{\mathrm{ACP}}=\left(\alpha_{\mathrm{Ca}^{2+}}\right)^{2}\left(\alpha_{\mathrm{HPO}_{4}^{2-}}\right)^{3}\right)$ with an estimated $K_{\text {sp }}(\mathrm{ACP})$ value of $\left(6.04 \times 10^{-4}\right)^{5}$ and $v_{\mathrm{m}}(\mathrm{ACP})=5.0 \times 10^{-23} \mathrm{~cm}^{3}$, as recently suggested by Habraken et al. ${ }^{25}$. The concentrations of ionic compounds and calculated $\sigma$ values are summarized in Supplementary Table 1. 
In situ X-ray scattering data collection and analysis. In situ SAXS data were collected during the mineralization of collagen at the Advanced Photon Source (APS, Sector 12 ID-B) at Argonne National Laboratory (Argonne, IL, USA). For the collagen mineralization, SBF-Ca and SBF-P were separately placed in $60 \mathrm{ml}$ syringes, then continuously flowed into the reactor at $0.11 \mathrm{ml} \mathrm{min}^{-1}$ per syringe, using a syringe pump. The volume of the solution in the reactor was $12.5 \mathrm{ml}$, giving $57 \mathrm{~min}$ of residence time, and maintaining the solution at a $\mathrm{pH}$ of $7.25 \pm 0.05$. A hot plate maintained the reactor at $37 \pm 1{ }^{\circ} \mathrm{C}$ (Supplementary Fig. 1b). Two frames holding two collagen matrices (a total of four samples in a reactor) were placed in the reactor for mineralization and temporarily $(\sim 2 \mathrm{~min})$ moved to the SAXS sample stage for analysis (Supplementary Fig. 1c). Samples were measured at intervals of $1-3 \mathrm{~h}$ during the mineralization, up to $15 \mathrm{~h}$. The distance from the sample to the SAXS detector was $3.6 \mathrm{~m}$, which provided a range of $0.0017-0.53 \AA^{-1}$ for the scattering vector, $q$. For each scan, the sample was exposed to a $14 \mathrm{keV} \mathrm{X-ray} \mathrm{beam}$ for $0.1 \mathrm{~s}$. With this experimental setup, we confirmed that homogeneous nucleation in $3 \times \mathrm{SBF}$ containing either 0 or $10 \mathrm{mg} \mathrm{l}^{-1}$ pAsp was not significant enough to be detectable by SAXS for up to $15 \mathrm{~h}^{23}$. Therefore, we concluded that the stability of the SBF solution was well maintained during the entire period of in situ measurements. The $2 \mathrm{D}$ scattering intensity counted by the detector ( $2 \mathrm{M}$ Pilatus) was averaged over the $q$ range along the radial direction to produce $1 \mathrm{D}$ scattering intensities, $I(q)$. Each obtained $I(q)$ was normalized by the incident beam intensity and calibrated on an absolute scale, using a reference glassy carbon standard sample ${ }^{45}$, and thus SAXS intensities collected from different measurements could be compared. Three different positions of each sample were analyzed, and their average $1 \mathrm{D}$ values were used for further analysis. The Modelling II tool of the IRENA package, written in IGOR Pro (WaveMetrics Inc.), was provided by APS and used to fit the SAXS pattern ${ }^{43}$.

In addition to SAXS, in situ WAXD analysis was conducted at APS sector 11ID-B to identify the phases of calcium phosphate minerals formed during collagen mineralization $\left(q>0.6 \AA^{-1}\right)$. For the data collection, five different positions of samples were exposed to a $58.66 \mathrm{keV} \mathrm{X-ray} \mathrm{beam} \mathrm{for} 2$ min each.

Ex situ sample analysis. For ex situ imaging of the mineralization, thin collagen films were prepared on glass slides to simulate the reaction occurring at the outermost surface of collagen matrices. To prepare a film, a droplet $(0.1 \mathrm{ml})$ of dissolved collagen $\left(12 \mathrm{mg} \mathrm{ml}^{-1}\right.$ of collagen in $0.5 \mathrm{mM} \mathrm{HCl}$ ) was evenly dispersed on a glass slide $\left(1 \times 1 \mathrm{~cm}^{2}\right)$ using a spin coater (Laurell WS-650MZ-23NPP, 5000 r.p.m. for $30 \mathrm{~s}$ ). Then the slide was placed in TES buffered saline for an hour to polymerize collagen fibrils. The mineralization of collagen films was also conducted in SBF solutions using the flow-through reaction system, as was done for in situ SAXS analysis. After the mineralization, these thin films were fixed in $100 \mathrm{mM}$ cacodylate buffer containing $2 \%$ paraformaldehyde and $2.5 \%$ glutaraldehyde. The films were then rinsed in a cacodylate buffer solution and dehydrated in successive ethanol baths $(30,50,70,90$, and $100 \%$ for $15 \mathrm{~min}$ each). For SEM imaging and energy dispersive spectroscopy (EDS) analysis, films were placed in SEM stubs and were sputter-coated with Au-Pd under Ar at $0.2 \mathrm{mbar}$ (Cressington 108) to increase conductivity, then imaged with a $10 \mathrm{kV}$ electron accelerating voltage at a $5-6 \mathrm{~mm}$ working distance (FEI Nova NanoSEM 230). EDS was calibrated by using $\mathrm{Cu}$ and Al standards with a measurement error of $\pm 1 \%$.

Data availability. Data supporting the findings of this study are available in the article and its Supplementary Information files, and are also available from the corresponding authors on request.

Received: 31 July 2017 Accepted: 16 January 2018

Published online: 06 March 2018

\section{References}

1. Jiang, Q. \& Ward, M. D. Crystallization under nanoscale confinement. Chem. Soc. Rev. 43, 2066-2079 (2014).

2. Page, A. J. \& Sear, R. P. Heterogeneous nucleation in and out of pores. Phys. Rev. Lett. 97, 065701 (2006).

3. Emmanuel, S., Anovitz, L. M. \& Day-Stirrat, R. J. Effects of coupled chemomechanical processes on the evolution of pore-size distributions in geological media. Rev. Mineral. Geochem. 80, 45-60 (2015).

4. Hedges, L. O. \& Whitelam, S. Patterning a surface so as to speed nucleation from solution. Soft Matter 8, 8624-8635 (2012).

5. Wang, Y. Nanogeochemistry: Nanostructures, emergent properties and their control on geochemical reactions and mass transfers. Chem. Geol. 378-379, 1-23 (2014).

6. Hamilton, B. D., Ha, J.-M., Hillmyer, M. A. \& Ward, M. D. Manipulating crystal growth and polymorphism by confinement in nanoscale crystallization chambers. Acc. Chem. Res. 45, 414-423 (2012).

7. Cantaert, B., Beniash, E. \& Meldrum, F. C. Nanoscale confinement controls the crystallization of calcium phosphate: relevance to bone formation. Chem. Eur. J. 19, 14918-14924 (2013).
8. Boskey, A. L. Mineralization of nones and teeth. Elements 3, 385-391 (2007)

9. Cantaert, B., Beniash, E. \& Meldrum, F. C. The role of poly(aspartic acid) in the precipitation of calcium phosphate in confinement. J. Mater. Chem. B 1, 6586-6595 (2013)

10. Wang, Y. et al. The predominant role of collagen in the nucleation, growth, structure and orientation of bone apatite. Nat. Mater. 11 (2012).

11. Alexander, B. et al. The nanometre-scale physiology of bone: steric modelling and scanning transmission electron microscopy of collagen-mineral structure. J. R. Soc. Interface 9, 1774-1786 (2012).

12. Orgel, J. P. R. O., Irving, T. C., Miller, A. \& Wess, T. J. Microfibrillar structure of type I collagen in situ. Proc. Natl Acad. Sci. USA 103, 9001-9005 (2006).

13. Hodge, A. J. \& Petruska, J. A. In Aspects of Protein Structure (ed. Ramachandran, G. N.) Page 13 (Academic Press, 1963)

14. Landis, W. J. \& Jacquet, R. Association of calcium and phosphate Ions with collagen in the mineralization of vertebrate tissues. Calcif. Tissue Int. $\mathbf{9 3}$, 329-337 (2013).

15. Landis, W. J., Song, M. J., Leith, A., McEwen, L. \& McEwen, B. F. Mineral and organic matrix interaction in normally calcifying tendon visualized in three dimensions by high-voltage electron microscopic tomography and graphic image reconstruction. J. Struct. Biol. 110, 39-54 (1993).

16. Nair, A. K., Gautieri, A., Chang, S.-W. \& Buehler, M. J. Molecular mechanics of mineralized collagen fibrils in bone. Nat. Commun. 4, 1724 (2013).

17. Olszta, M. J. et al. Bone structure and formation: A new perspective. Mater. Sci. Eng. R. 58, 77-116 (2007).

18. Masic, A. et al. Osmotic pressure induced tensile forces in tendon collagen. Nat. Commun. 6, 5942 (2015).

19. Omelon, S. et al. Control of vertebrate skeletal mineralization by polyphosphates. PLoS. One. 4, e5634 (2009).

20. Nudelman, F. et al. The role of collagen in bone apatite formation in the presence of hydroxyapatite nucleation inhibitors. Nat. Mater. 9, 1004-1009 (2010).

21. Chen, L., Jacquet, R., Lowder, E. \& Landis, W. J. Refinement of collagen-mineral interaction: A possible role for osteocalcin in apatite crystal nucleation, growth and development. Bone 71, 7-16 (2015).

22. Landis, W. J. et al. Mineralization of collagen may occur on fibril surfaces: Evidence from conventional and high-voltage electron microscopy and threedimensional Imaging. J. Struct. Biol. 117, 24-35 (1996).

23. Kim, D., Lee, B., Thomopoulos, S. \& Jun, Y.-S. In situ evaluation of calcium phosphate nucleation kinetics and pathways during intra and extrafibrillar mineralization of collagen matrices. Cryst. Growth Des. 16, 5359-5366 (2016).

24. Dey, A. et al. The role of prenucleation clusters in surface-induced calcium phosphate crystallization. Nat. Mater. 9, 1010-1014 (2010).

25. Habraken, W. J. E. M. et al. Ion-association complexes unite classical and nonclassical theories for the biomimetic nucleation of calcium phosphate. Nat. Commun. 4 (2013).

26. Gebauer, D. \& Cölfen, H. Prenucleation clusters and non-classical nucleation Nano Today 6, 564-584 (2011)

27. Jee, S.-S., Thula, T. T. \& Gower, L. B. Development of bone-like composites via the polymer-induced liquid-precursor (PILP) process. Part 1: Influence of polymer molecular weight. Acta Biomater. 6, 3676-3686 (2010).

28. De Yoreo, J. J. \& Vekilov, P. G. Principles of crystal nucleation and growth Rev. Mineral. Geochem. 54, 57-93 (2003).

29. De Yoreo, J. J., Waychunas, G. A., Jun, Y.-S. \& Fernandez-Martinez, A. In situ investigations of carbonate nucleation on mineral and organic surfaces. Rev. Mineral. Geochem. 77 (2013).

30. Jun, Y.-S., Kim, D. \& Neil, C. W. Heterogeneous nucleation and growth of nanoparticles at environmental interfaces. Acc. Chem. Res. 49, 1681-1690 (2016).

31. Li, Q., Fernandez-Martinez, A., Lee, B., Waychunas, G. A. \& Jun, Y.-S Interfacial energies for heterogeneous nucleation of calcium carbonate on mica and quartz. Environ. Sci. Technol. 48, 5745-5753 (2014).

32. Gebauer, D., Kellermeier, M., Gale, J. D., Bergstrom, L. \& Colfen, H. Prenucleation clusters as solute precursors in crystallisation. Chem. Soc. Rev. $\mathbf{4 3}$, 2348-2371 (2014)

33. Meldrum, F. C. \& Sear, R. P. Now you see them. Science 322, 1802-1803 (2008).

34. De Yoreo, J. J. et al. Crystallization by particle attachment in synthetic, biogenic, and geologic environments. Science 349 (2015).

35. Koutsoukos, P. G. \& Nancollas, G. H. The mineralization of collagen in vitro. Colloids Surf. 28, 95-108 (1987).

36. Ito, S., Saito, T. \& Amano, K. In vitro apatite induction by osteopontin: interfacial energy for hydroxyapatite nucleation on osteopontin. J. Biomed. Mater. Res., Part A 69, 11-16 (2004).

37. Koutsopoulos, S., Paschalakis, P. C. \& Dalas, E. The calcification of elastin in vitro. Langmuir 10, 2423-2428 (1994).

38. $\mathrm{Xu}, \mathrm{Z}$. et al. Small molecule-mediated control of hydroxyapatite growth: Free energy calculations benchmarked to density functional theory. J. Comput. Chem. 35, 70-81 (2014). 
39. Delgado-López, J. M. et al. Crystal size, morphology, and growth mechanism in bio-inspired apatite nanocrystals. Adv. Funct. Mater. 24, 1090-1099 (2013).

40. Na, H. G. et al. One-to-one correspondence growth mechanism of gourd-like SiOx nanotubes. Cryst Growth Des. 16 (2016).

41. $\mathrm{Xu}, \mathrm{Z}$. et al. Molecular mechanisms for intrafibrillar collagen mineralization in skeletal tissues. Biomaterials 39, 59-66 (2015).

42. Li, T., Senesi, A. J. \& Lee, B. Small angle X-ray scattering for nanoparticle research. Chem. Rev. 116, 11128-11180 (2016).

43. Ilavsky, J. \& Jemian, P. R. Irena: tool suite for modeling and analysis of smallangle scattering. J. Appl. Crystallogr. 42, 347-353 (2009).

44. Fratzl, P. Small-angle scattering in materials science-a short review of applications in alloys, ceramics and composite materials. J. Appl. Crystallogr. 36, 397-404 (2003).

45. Zhang, F. et al. Glassy carbon as an absolute intensity calibration standard for small-angle scattering. Metall. Mater. Trans. A 41, 1151-1158 (2010).

46. Mahamid, J. et al. Mapping amorphous calcium phosphate transformation into crystalline mineral from the cell to the bone in zebrafish fin rays. Proc. Natl. Acad. Sci. USA 107, 6316-6321 (2010).

47. Betts, F. \& Posner, A. S. An X-ray radial distribution study of amorphous calcium phosphate. Mater. Res. Bull. 9, 353-360 (1974).

48. Posner, A. S. \& Betts, F. Synthetic amorphous calcium phosphate and its relation to bone mineral structure. Acc. Chem. Res. 8, 273-281 (1975).

49. Grynpas, M. D., Bonar, L. C. \& Glimcher, M. J. Failure to detect an amorphous calcium-phosphate solid phase in bone mineral: A radial distribution function study. Calcif. Tissue Int. 36, 291-301 (1984).

50. Jäger, C., Welzel, T., Meyer-Zaika, W. \& Epple, M. A solid-state NMR investigation of the structure of nanocrystalline hydroxyapatite. Magn. Reson. Chem. 44, 573-580 (2006)

51. Beaucage, G., Kammler, H. K. \& Pratsinis, S. E. Particle size distributions from small-angle scattering using global scattering functions. J. Appl. Crystallogr. 37, 523-535 (2004)

52. Wu, Y.-T. \& Grant, C. Effect of chelation chemistry of sodium polyaspartate on the dissolution of calcite. Langmuir 18, 6813-6820 (2002).

53. Pasteris, J. D., Wopenka, B. \& Valsami-Jones, E. Bone and tooth mineralization: Why apatite? Elements 4, 97-104 (2008).

54. Kerschnitzki, M. et al. Bone mineralization pathways during the rapid growth of embryonic chicken long bones. J. Struct. Biol. 195, 82-92 (2016).

55. Buckton, G. \& Beezer, A. E. The relationship between particle size and solubility. Int. J. Pharm. 82, R7-R10 (1992).

56. Scherer, G. Crystallization in pores. Cem. Concr. Res. 29, 1347-1358 (1999).

57. Anduix-Canto, C. et al. Effect of nanoscale confinement on the crystallization of potassium ferrocyanide. Cryst. Growth Des. 16, 5403-5411 (2016).

58. Tas, A. C. The use of physiological solutions or media in calcium phosphate synthesis and processing. Acta Biomater. 10, 1771-1792 (2014).

59. Boskey, A. L. Noncollagenous matrix proteins and their role in mineralization. Bone Miner. 6, 111-123 (1989).

60. Zhang, H., Gilbert, B., Huang, F. \& Banfield, J. F. Water-driven structure transformation in nanoparticles at room temperature. Nature 424, 1025-1029 (2003).

61. Boyle, J. J. et al. Simple and accurate methods for quantifying deformation, disruption, and development in biological tissues. J. R. Soc. Interface. 11 (2014).

62. Ohtsuki, C., Kokubo, T. \& Yamamuro, T. Mechanism of apatite formation on $\mathrm{CaOSiO}_{2} \mathrm{P}_{2} \mathrm{O}_{5}$ glasses in a simulated body fluid. J. Non-Cryst. Solids 143, 84-92 (1992).

63. Gamble, J. L. Chemical Anatomy Physiology and Pathology of Extracellular Fluid: A Lecture Syllabus. Sixth edn (Harvard University Press, Cambridge, MA, 1967).
64. Lu, X. \& Leng, Y. Theoretical analysis of calcium phosphate precipitation in simulated body fluid. Biomaterials 26, 1097-1108 (2005).

65. Davies, C. W. Ion Association (Butterworths, Washington, DC, 1962).

66. McDowell, H., Gregory, T. \& Brown, W. Solubility of $\mathrm{Ca}_{5}\left(\mathrm{PO}_{4}\right)_{3} \mathrm{OH}$ in the system $\mathrm{Ca}(\mathrm{OH})_{2}-\mathrm{H}_{3} \mathrm{PO}_{4}-\mathrm{H}_{2} \mathrm{O}$ at 5, 15, 25, and $37^{\circ} \mathrm{C}$. J. Res. Nat. Bur. Stand. A 81, 273-281 (1977)

67. Boistelle, R. \& Lopez-Valero, I. Growth units and nucleation: The case of calcium phosphates. J. Cryst. Growth 102, 609-617 (1990).

\section{Acknowledgements}

We acknowledge Dr. Jill Pasteris, Dr. Alix C. Deymier, Dr. Guy Genin, and Dr. Tyrone Daulton for helpful discussions. We thank Prof. James Ballard for carefully reviewing the manuscript. The project was supported by the National Science Foundation (DMR1608545 and DMR-1608554). The Nano Research Facility and the Institute of Materials Science \& Engineering at Washington University in St. Louis provided their facilities for the experiments. Use of the Advanced Photon Source (sectors 12-ID-B and 11-ID-B) at Argonne National Laboratory was supported by the U.S. Department of Energy, Office of Science, Office of Basic Energy Sciences, under Contract No. DE-AC02-06CH11357.

\section{Author contributions}

D.K. and Y.-S.J. conducted most of the experiments and wrote the manuscript. B.L. contributed to SAXS data collection and analysis. S.T. provided collagen preparation and treatments. All authors discussed the experimental design and the results, and collectively revised the manuscript.

\section{Additional information}

Supplementary Information accompanies this paper at https://doi.org/10.1038/s41467 018-03041-1.

Competing interests: The authors declare no competing financial interests.

Reprints and permission information is available online at http://npg.nature.com/ reprintsandpermissions/

Publisher's note: Springer Nature remains neutral with regard to jurisdictional claims in published maps and institutional affiliations.

Open Access This article is licensed under a Creative Commons Attribution 4.0 International License, which permits use, sharing, adaptation, distribution and reproduction in any medium or format, as long as you give appropriate credit to the original author(s) and the source, provide a link to the Creative Commons license, and indicate if changes were made. The images or other third party material in this article are included in the article's Creative Commons license, unless indicated otherwise in a credit line to the material. If material is not included in the article's Creative Commons license and your intended use is not permitted by statutory regulation or exceeds the permitted use, you will need to obtain permission directly from the copyright holder. To view a copy of this license, visit http://creativecommons.org/ licenses/by/4.0/

(C) The Author(s) 2018 